\title{
Heart Failure With Preserved Ejection Fraction: A Perioperative Review
}

\author{
Sasha K. Shillcutt, MD, MS, FASE ${ }^{1}$, M. Megan Chacon, MD, Tara R. Brakke, MD, FASE, Ellen \\ K. Roberts, MD, Thomas E. Schulte, MD, and Nicholas Markin, MD, FASE \\ University of Nebraska Medical Center, Department of Anesthesiology, Omaha, NE
}

\begin{abstract}
HEART FAILURE (HF) with preserved ejection fraction (HFpEF) presents significant challenges for anesthesiologists. Nearly $50 \%$ of patients presenting with HF have HFpEF, defined as having clinical HF with a left ventricular ejection fraction (LVEF) $>50 \%$ and abnormal left ventricular diastolic dysfunction (LVDD). ${ }^{1-6}$ Even though HFpEF is a strong predictor of negative postoperative outcomes, it often is difficult to diagnose and the management strategies are unclear. Although there have been specific articles published with regard to the echocardiography descriptors, diagnosis, and relationship of LVDD with HF, the authors believed it was timely to present a review article for clinicians on the subject of perioperative HFpEF. This article reviews what is known about the pathogenesis, diagnosis, management options, and implications of HFpEF in the perioperative arena.
\end{abstract}

\section{Definitions: HFpEF, HF With Reduced Ejection Fraction, and LVDD}

$\mathrm{HF}$ in general is defined as the inability of the heart to function sufficiently to meet the metabolic demands of the body. The diagnosis applies to both phenotypes of HF-HF with reduced ejection fraction (HFrEF) and HFpEF. Both types of HF have similar signs, symptoms, morbidity, and mortality; however, the pathophysiology underlying HFpEF is illdefined because no single abnormality exists that explains the mechanism of disease. The presence of HF symptoms and the preservation of systolic function often lead to HFpEF, which, until recently, has been referred to as "diastolic HF". Although the pathologic underpinnings may seem ill-defined, one common attribute in HFpEF is diastolic filling abnormalities of the left ventricle. LVDD is characterized by a stiff left ventricle, with decreased compliance and impaired relaxation, which leads to increased end-diastolic pressure.

\section{Prevalence and Incidence of HFpEF}

Due to rapid aging and a longer life expectancy of both sexes, the incidence of HFpEF is growing in the general population. An increasing presence of comorbidities associated with aging is key to understanding the increase in HFpEF prevalence. HFpEF patients generally are older, more hypertensive, obese, diabetic, and likely to have atrial fibrillation compared with patients with HFrEF. ${ }^{1,7}$ A follow-up to the Prevention of Renal and Vascular Endstage

\footnotetext{
${ }^{1}$ Address reprint requests to Sasha K. Shillcutt, MD, MS, FASE, University of Nebraska Medical Center, 984455 Nebraska Medical Center, Omaha, NE 68198-4455. sshillcu@unmc.edu (S.K. Shillcutt).
} 
Disease (PREVEND) study that included 8,592 patients demonstrated the association between age, history of atrial fibrillation, and prevalence of HFpEF. ${ }^{8}$ This community-based cohort trial identified all cases of new-onset HF during 11 years of follow-up and identified risk factors for HFrEF and HFpEF. The age and sex-specific prevalence of HFpEF was $1 \%$ in women and $0 \%$ in men between ages 25 to 49 years, but increased to $8 \%$ to $10 \%$ in women and $4 \%$ to $6 \%$ in men ages $\$ 80$ years. ${ }^{8}$ This trend toward increased rates of $\mathrm{HFpEF}$ with increasing age was supported in other studies, including one that demonstrated that up to $59 \%$ of all patients $>85$ years had HFpEF. 9,10 Annual mortality ranges from $10 \%$ to $30 \%$, with nearly $60 \%$ of HFpEF patients dying from cardiovascular causes. ${ }^{7}$ However, noncardiovascular deaths constitute a higher proportion of cause of death in HFpEF compared with $\mathrm{HFrEF}^{7}$

\section{Pathophysiology of HFpEF}

$\mathrm{HFpEF}$, like other clinical presentations of $\mathrm{HF}$, is a syndrome comprising various underlying etiologies and comorbid conditions that lead to the presence of both symptoms and signs consistent with the diagnosis. ${ }^{9}$ The cause of HFpEF is multifactorial, but the consistent underlying aspects are similar: a proinflammatory state perpetuated by one or more of several disease states leads to inflammation within the coronary microvasculature and its endothelium. Endothelial dysfunction leads to reductions in available nitric oxide and activity of protein kinase $\mathrm{G}$ (PKG). The reduced activity of PKG leads to dysregulation of prohypertrophic pathways and leads to overphosphorylation of titin. ${ }^{3,10-13}$ This causes stiffening of the myocardium and interstitial fibrosis that contribute to the LVDD of the myocardium and results in the presentation of clinical symptoms. The proinflammatory state is attributed to increased central autonomic tone via a central chemoreflex pathway that is responsible for causing diastolic dysfunction and imbalance between the sympathetic and parasympathetic setpoint. ${ }^{14}$ Pressure-volume loops show an upward or leftward shift of the LV end-systolic pressure volume relationship, reflecting increased end-systolic myocardial stiffness. Patients with HFpEF typically have normal LV chamber size and greater wall thickness (concentric remodeling), resulting in lower brain natriuretic peptide (BNP) levels. ${ }^{1,4} \mathrm{~A}$ key determinant of BNP release is diastolic wall stress, thus these levels often are normal in patients with HFpEF. Although LVEF is normal at rest in HFpEF, it does not increase appropriately during stress. ${ }^{15}$ This decreased LV contractility may be related to abnormalities in calcium handling, beta-adrenergic signaling, myocardial energetics, or tissue perfusion reserve. ${ }^{1}$ Ventricular and vascular stiffening are increased, which lead to an increase in blood pressure. This strains the heart and further impairs diastolic relaxation, thus leading to dramatic increases in filling pressures during stress and exercise. ${ }^{4}$ This also leads to larger-than-expected decreases in systolic blood pressure with decreases in volume. See Table 1 for additional information.

\section{Preclinical HFpEF: LVDD With a Preserved EF}

LVDD, a requirement for the clinical diagnosis of HFpEF, has been associated with poor outcomes in both cardiac and noncardiac surgery. ${ }^{16-23}$ The presence of both preoperative LVDD on transthoracic echocardiography (TTE) and the identification of LVDD intraoperatively are predictors of worse perioperative outcomes. ${ }^{23}$ There were several studies 
whose cohort included normal EF and significant LVDD—at least grade II; however, more typically grade-III diastolic dysfunction — which has been referred to as "preclinical HFpEF". It is important to note, however, that these patients were not classified as having $\mathrm{HF}$; thus, the majority of literature on clinical studies in the perioperative arena is on the preclinical findings of HFpEF. A summary of these studies are provided in Table 2.

Although inhaled anesthetic gases and other anesthetic agents that reduce afterload have a beneficial effect on diastolic function, the impact of anesthetics, surgical manipulation, and hemodynamic instability on changes in perioperative LVDD and how this relates to HFpEF need further clarification and study.

\section{Association with Mortality and Major Adverse Cardiac Events}

Kaw et al performed a meta-analysis on 12 prospective and retrospective clinical studies examining preoperative LVDD and mortality and major adverse cardiac events (MACE) in cardiac surgery perioperatively. ${ }^{21}$ A subgroup analysis was performed on patients with LVDD, comparing patients with normal LVEF, defined as $>40 \%$, and patients with reduced LVEF ( $<40 \%)$. Mortality was shown to increase with preoperative grade-III LVDD. ${ }^{21}$ Dalén et al studied 41,906 patients undergoing coronary artery bypass grafting (CABG) and found that all-cause mortality was statistically higher long term for patients with HF independent of EF. ${ }^{27}$ Increasing grade of LVDD also was predictive of a greater incidence of adverse events, including stroke, renal failure, prolonged ventilation, deep sternal wound infection, or redo surgery. ${ }^{17,19}$ Intraoperatively, LVDD has been shown to predict increased difficulty in weaning from cardiopulmonary bypass. ${ }^{24,30}$ Both studies observed an association between moderate and severe LVDD, with difficulty in separating from cardiopulmonary bypass and hemodynamic instability requiring more inotropic and mechanical support. However, LVEF was not used in the stratification of these investigations, thus larger studies are necessary to verify whether preclinical HFpEF increases perioperative hemodynamic instability and, specifically, MACE. Investigators in many of the studies defined MACE differently, including myocardial ischemia, arrhythmias, cardiovascular death, clinical pulmonary edema, and HF; they also included various LVDD measurement techniques and definition of parameters. ${ }^{19,26,29,31}$

\section{Preoperative Risk Evaluation and HFpEF}

Currently, neither the Society of Thoracic Surgeons risk score nor the European System for Cardiac Operative Risk Evaluation (EuroSCORE) uses preclinical signs of HFpEF to riskassess patients preoperatively. Patients who report symptoms consistent with congestive HF within 2 weeks before a procedure are risk stratified as part of the Society of Thoracic Surgeons risk score, but LVDD is not part of that assessment. Afilalo et $\mathrm{al}^{23}$ and Sastry et $\mathrm{al}^{16}$ found that preoperative LVDD was predictive of post-CABG outcomes, including inhospital mortality and major morbidity. This poses the question of whether screening patients with preclinical signs of HFpEF improves risk stratification of surgical patients.

Perioperative LVDD has a significant association with postoperative congestive HF and pulmonary edema, both of which may be clinical signs of HFpEF. Cho et al evaluated perioperative LVDD in 692 patients undergoing low-to-moderate risk noncardiac surgery, 
including 166 with pulmonary edema postoperatively. Of those with edema, preoperative TTE showing an E/e' ratio >15 increased the risk of postoperative pulmonary edema and MACE. ${ }^{29}$ Increases in pulmonary edema and longer intensive care unit and hospital stays also have been associated with an E/e' ratio $>12 .{ }^{28}$ Matyal et al found a significantly longer hospitalization in patients with perioperative LVDD, including longer hospitalization within the HFpEF patient subgroup. ${ }^{17}$

\section{Diagnosis of HFpEF}

The clinical diagnosis of HF requires the presence of symptoms consistent with those listed in Table $3 .^{32}$ Differentiation of noncardiac from cardiac causes can be difficult, especially when the clinical symptoms are vague and nondescript, as HF patients commonly report to their anesthesiologist. Shortness of breath, exercise intolerance, and fatigue can have multiple causes, but these symptoms along with findings consistent with signs of HFpEF lead to the diagnosis. These symptoms occur because of physiologic alterations in cardiac function, specifically diastolic filling and the diastolic pressures required for ventricular filling.

\section{Diagnostic Criteria for HFpEF}

Diagnostic criteria for HFpEF are important because the literature has varied in the description of HFpEF and in the inclusion criteria of studies published in the last decade. Guidelines by both the American College of Cardiologists and American Heart Association (ACC/AHA) and the European Society of Cardiology (ESC) provide direction with specific diagnostic criteria. The ACC/AHA use the following diagnostic criteria: (1) the presence of HF symptoms in the setting of a preserved EF, (2) LVEF $250 \%$, and (3) abnormal LV diastolic function. ${ }^{33}$ This is referred to by the ACC/AHA as "diastolic HF", requiring the identification of LVDD and the exclusion of other noncardiac causes of the patient's reported symptoms. In the perioperative period the identification of symptoms consistent with HF is crucial to making the appropriate diagnosis because symptomatic LVDD is associated with increased cardiac events and associated mortality. Specifically examining HFpEF and CABG, the HFpEF and HFrEF groups reached similar mortality rates at 5-year follow-up, with a 33\% all-cause mortality compared with $15 \%$ in the normal LV systolic and diastolic group. ${ }^{31}$ It is recommended that a comprehensive Doppler evaluation be completed during echocardiography, especially in the setting of known or suspected HF. ${ }^{15}$ Frequently reported symptoms of HF include dyspnea, exercise intolerance, and fluid retention. ${ }^{6,34}$ These clinical findings are summarized in Table 3.

The ACC/AHA guidelines are nonspecific and emphasize clinical history but lack of other identifiable causes of HF to make the diagnosis. However, the ESC guidelines for HF include more defined diagnostic criteria for HFpEF. The ESC definition stresses the importance of appropriate history gathering when diagnosing HFpEF. The diagnosis, if present, should be made preoperatively because HF is a risk factor for poor outcomes after noncardiac surgery. ${ }^{35}$ Population studies have demonstrated a significant prevalence of LVDD among the general population; therefore, the findings of diastolic filling 
abnormalities alone should not portend a diagnosis of HFpEF. ${ }^{36}$ It is yet to be determined whether LVDD is presymptomatic HF in the setting of preserved systolic function. ${ }^{35}$

The ESC guidelines focus on the following main points: (1) symptoms of HF, (2) LVEF $250 \%$, (3) LV end-diastolic volume $<97 \mathrm{~mL} / \mathrm{m}^{2}$, and (4) the presence of LVDD. ${ }^{35}$ The diagnosis of LVDD can be difficult, and there are various suggested diagnostic methods, including Doppler mitral inflow velocities and mitral inflow velocity ratios between the early (E-wave) and atrial (A-wave) diastolic filling periods. The ratio of the E-wave to the tissue Doppler velocities of the mitral annulus $\left(\mathrm{e}^{\prime}\right)$ can be used to suggest elevated diastolic filling pressures. ${ }^{35}$ In addition, the presence of enlarged left atrial volume index and elevations in BNP or N-terminal pro-BNP (NTproBNP) can indicate LVDD and elevated LV filling pressures. To this point, the ESC's recommendations do not specifically indicate the preferred method of determining LVDD but recommend that the evaluation performed should focus on evidence of structural and Doppler findings that confirm the presence of LVDD is consistent with the diagnosis of HFpEF. A summary of both the ACC/AHA and the ESC definitions and diagnostic criteria are presented in Table 4.

\section{Perioperative Evaluation of Diastolic Function}

Guidelines by the American Society of Echocardiography and investigations conducted during the perioperative setting can guide the anesthesiologist on how best to diagnose the presence of LVDD. ${ }^{37,38}$ Specific to diastolic function and its evaluation, the literature is predominantly based on TTE findings. ${ }^{38,39}$ The underlying cause of impairment in diastolic relaxation can be linked to abnormal calcium handling. ${ }^{40}$ Although the anesthetized patient has been shown to have preserved myocardial performance measures through maintenance of ventriculoarterial coupling when exposed to both volatile and intravenous anesthetic agents, ${ }^{41,42}$ abnormal vascular tone from external or native sympathetic-driven activation can lead to elevations in left ventricular end-diastolic pressure and left atrial pressure. ${ }^{43}$ To further complicate the difficulty associated with the performance of intraoperative evaluation of LVDD, there are data to suggest that abnormal calcium homeostasis in the anesthetized state may affect the diastolic filling of the left ventricle. ${ }^{44}$ The major measurements of diastolic function are (1) mitral inflow velocities and ratios, (2) tissue Doppler velocities and the ratio of E-wave velocities to $\mathrm{e}^{\prime}$ velocities, and (3) the pattern of venous flow into the left atrium from the pulmonary veins. ${ }^{38,39,45} \mathrm{~A}$ revised perioperative echocardiographic approach to quantification of diastolic function was published by Mahmood et al in 2012. ${ }^{46}$ The significance of this work was to dichotomize the perioperative state into reduced compliance/ high filling pressures (LVDD) and normal compliance using the lateral tissue Doppler velocity in which tissue Doppler imaging was either $\geq 10 \mathrm{~cm} / \mathrm{sec}$ or $<10 \mathrm{~cm} / \mathrm{sec}^{4}{ }^{47}$ One limitation of this approach was that it required the performance of TTE to accurately measure the indexed size of the left atrium.

In regard to these prior studies, the authors suggest that the performance of lateral tissue Doppler measurements, dividing the perioperative state into $\geq 10 \mathrm{~cm} / \mathrm{sec}$ and $<10 \mathrm{~cm} / \mathrm{sec}$ and determining E/ $\mathrm{e}^{\prime}$ ratios and E/A ratios, is helpful in the classification and diagnosis of LVDD. ${ }^{39}$ Clearly, the direct application of the TTE-derived guidelines does not allow for accurate classification of many perioperative patients. ${ }^{39}$ Using these studies to guide clinical 
practice and the criteria summarized in Table 5, the authors classify patients into the following groups: normal, impaired relaxation with normal-range LV end-diastolic filling pressures, and LVDD with elevated diastolic filling pressures. ${ }^{47}$

The diagnostic criteria, as seen on echocardiography, can be demonstrated through routine two-dimensional analysis and measurements and using a comprehensive Doppler evaluation approach to measuring tissue and blood Doppler-derived flow. In an example of HFpEF, Figure 1 demonstrates normal systolic function in the form of preserved systolic function. Figure 2 demonstrates the common Doppler findings pertaining to tissue Doppler velocities, mitral inflow velocities, and pulmonary venous flow consistent with LVDD and elevated left ventricular end-diastolic pressure, requisite findings of HFpEF. These images, in conjunction with patient history consistent with HF symptoms, demonstrate the 4 diagnostic criteria as established by the ESC.

\section{Testing in the Perioperative Setting}

In addition to echocardiographic assessments, which can be performed in the preoperative or intraoperative period, other assessments are suggested to be beneficial in the assessment of HF. These assessments include laboratory analysis of biomarkers specific to elevated intracardiac filling pressures, echocardiography stress testing, cardiac magnetic resonance imaging, and right-and left-heart catheterization. ${ }^{35,48}$ During the perioperative period, some of these studies such as magnetic resonance imaging or heart catheterization have limited utility due to time constraints. With appropriate lead time on the identification of HF symptoms, routine TTE for anatomic and functional analysis is considered a class-I indication in the ACC/AHA and ESC guidelines. ${ }^{34,35}$ If the TTE study reveals normal systolic and diastolic function but the clinical suspicion is high, the performance of a diastolic stress test may elucidate the presence of HFpEF in patients who report exercise intolerance but do not have abnormal LVDD on resting echocardiography. ${ }^{49-51}$

Recommendations for diastolic stress testing should occur when resting evaluation of LV diastolic function is indeterminate. Exercise is preferred over dobutamine stress testing and is considered positive for stress-induced diastolic dysfunction if all of the 3 following measurements are present: average E/ $\mathrm{e}^{\prime}>14$, peak tricuspid regurgitation velocity (TR) $>2.8$ $\mathrm{m} / \mathrm{sec}$, and septal $\mathrm{e}^{\prime}<7 \mathrm{~cm} / \mathrm{sec}^{51}{ }^{5}$ Increases of E/e' $>14$ have a $90 \%$ sensitivity and $71 \%$ sensitivity for HFpEF and suggest that exercise diastolic stress testing may rule out HFpEF. 52 When requesting the performance of this study specifically for diastolic evaluation, it is critical to appropriately request the specific information from the stress test because diastolic stress evaluation is not routine at all centers.

Biomarker testing is another potentially helpful diagnostic test during the perioperative period. ${ }^{49}$ Although there are new potential markers being discovered (IGFBP7, copeptin, MR-proADM, ST2, and galectin-3), the suspicion of HF and elevated filling pressures can be further supported through BNP or NTproBNP testing. ${ }^{53}$ Elevated biomarkers can be useful in both the diagnosis and after medical interventions for improved diastolic filling. Measurement of BNP or NTproBNP in the setting of clinical uncertainty or after treatment to assess changes is considered a class-I indication in the ACC/AHA guidelines and a classIIa indication in the ESC guidelines. ${ }^{34,35}$ In the preoperative evaluation, assessment of HF 
with BNP may prove useful in risk stratification for these patients because HF itself is an independent risk for perioperative cardiovascular morbidity and mortality. The ESC guidelines indicate that BNP $\geq 200 \mathrm{pg} / \mathrm{mL}$ or NTproBNP $\geq 20 \mathrm{pg} / \mathrm{mL}$ is consistent with HF. ${ }^{5}$ Even though using BNP and NTproBNP can be helpful, there are instances in which the BNP result can be inaccurate. In the setting of atrial fibrillation, the level of BNP can be elevated in the absence of significant LVDD and elevated diastolic filling pressures. ${ }^{54,55}$ Other signs of elevated diastolic filling pressures should be used to confirm HFpEF, which can be difficult to determine in the presence of atrial fibrillation as well. Because atrial fibrillation will result in the loss of an A-wave on transmitral Doppler inflow, other methods such as E/e' and pulmonary venous flow patterns should be used to assess diastolic function.

Obesity is a commonly associated comorbid condition with HFpEF, and it is speculated that increased body mass itself is an additional contributing factor to the symptoms experienced when obesity and HFpEF coexist. ${ }^{6,56}$ A confounder in the evaluation of an obese patient with suspected HFpEF is that obesity is associated with lower-than-expected BNP levels for the degree of diastolic pressure elevation and HF symptoms. ${ }^{15,57,58}$ BNP results that do not reach the cutoff for $\mathrm{HFpEF}$ in very obese patients, or in some patients without baseline values, should not rule out the presence of diastolic HF if clinical suspicions are high.

\section{Perioperative Evaluation Strategy}

The diagnosis of HF first requires the suspicion of HF to elicit historic symptoms consistent with the diagnosis. In the perioperative period the preanesthesia evaluation already is well positioned to be used to discover these symptoms because dyspnea, exercise tolerance, respiratory symptoms, and chest pain commonly are assessed. ${ }^{59}$ When patients report poor exercise tolerance, dyspnea with exertion, or increasing fluid retention, the performance of a resting TTE is an important first step to determine LV systolic and diastolic function. When these studies are ambiguous, additional testing, such as BNP or diastolic stress testing, can be helpful. Diastolic stress testing is recommended when resting evaluation of LV diastolic function is indeterminant.

When performed with the patient under general anesthesia, Doppler echocardiography filling parameters should emphasize the lateral mitral annular tissue Doppler velocity because that is the most load-independent measure in the diastolic evaluation, and even though tissue Doppler is not completely load-independent, it is recognized as the least affected parameter with changes in preload. ${ }^{38}$ The presence of abnormal diastolic filling patterns assists the authors in perioperative management by augmenting the allowable fluid administration and supports the early use of inotropic support for alterations in hemodynamics as opposed to additional fluid administration to avoid the consequences of pulmonary edema and vascular overload. In the event the patient is found to have elevated diastolic filling pressures, the first-line intervention for hypotension often is inotropic support and not fluid administration to prevent further increases in left atrial pressure, which may result in pulmonary edema. 


\section{Management Options in HFpEF}

As previously stated, identifying patients who have or are at risk for HFpEF is the first step in perioperative management. ${ }^{60}$ It is important to optimize comorbid conditions before surgery, including aggressive control of chronically uncontrolled hypertension and avoidance of significant hyperglycemia. Sinus rhythm should be restored if possible, and the atrial fibrillation rate should be controlled. Continuous positive airway pressure devices should be worn for patients with obstructive sleep apnea. ${ }^{61}$ Intraoperative echocardiography is the most effective tool to monitor increasing grades of LVDD. ${ }^{61}$ Furthermore, it has been demonstrated that the grade of severity of LVDD can change throughout the course of general anesthesia, and decisions regarding fluid and medication management should not be made solely based on the preoperative assessment. ${ }^{48}$ Fluid administration in a patient with LVDD may be necessary to improve LV filling, but excessive fluid administration may result in increased end-diastolic pressure that may exacerbate pulmonary venous congestion, leading to pulmonary edema. ${ }^{61}$

Large trials using angiotensin-converting enzyme inhibitors and angiotensin-II receptor blockers have yielded disappointing results and have shown neutral outcomes. ${ }^{62} \mathrm{~A}$ review of the prospective clinical studies of renin-angiotensin (RAS) inhibitors (CHARM-Preserved, I-Preserve, PEP-CHF) have shown no clear benefit with regard to mortality or hospitalization for HF. ${ }^{63}$ Both beta-blocker and spironolactone trials have yielded inconclusive results. ${ }^{62}$ The Treatment of Preserved Cardiac Function HF with an Aldosterone Antagonist trial was a randomized, double-blind trial that examined 3,445 patients with $\mathrm{HFpEF}$ assigned to spironolactone or placebo over a 3-year period. No difference in death from cardiovascular causes, cardiac arrest, or hospitalization for HF was found between the 2 groups. ${ }^{64} \mathrm{~A}$ recent meta-analysis examined the effect of beta-blocker therapy on mortality and hospitalization for HF in HFpEF patients. Fifteen observational studies and 2 randomized controlled trials (RCTs) were included. In the observational studies, beta-blocker therapy was associated with lower mortality but not HF hospitalization, whereas beta-blocker therapy was not associated with a decrease in mortality or morbidity in the RCTs, indicating the need for additional trials. ${ }^{65}$ Experimental data have suggested that phosphodiesterase- 5 inhibitors may enhance cardiovascular function by dilation of the pulmonary vascular bed, enhancement of right ventricular contractility, reduction of ventricular interdependence, and prevention of cardiomyocyte remodeling and, therefore, might be of promise in HFpEF. However, a randomized, double-blind, placebo-controlled clinical trial of 216 patients with stable HFpEF did not result in significant improvement in exercise capacity or clinical status after 24 weeks of sildenafil administration. ${ }^{66}$

Currently, no treatment has been proven to reduce morbidity and mortality in patients with HFpEF. ${ }^{67}$ Comorbid conditions should be treated optimally. Most medications typically avoided in HFrEF also should be avoided in HFpEF, with the exception of verapamil and diltiazem. ${ }^{68}$ Exercise training improves quality of life and symptoms. ${ }^{68}$ Significantly fewer exercise therapy trials exist for the HFpEF population than for patients with HFrEF, but a meta-analysis indicated that moderate-intensity exercise programs elicited improvements in LVDD and health-related quality of life. ${ }^{69}$ Options for management are included in Table 6. 


\section{Future Directions: The New HFpEF Paradigm}

Arterial hypertension long has been believed to cause chronically elevated afterload, leading to ventricular remodeling and resulting in HFpEF. Recent work by Paulus et al proposed a new paradigm for the sequence of events that leads to myocardial remodeling and dysfunction. ${ }^{10}$ Their paradigm focused on the comorbidities obesity, diabetes mellitus, chronic obstructive pulmonary disease, and hypertension, all of which are capable of causing the systemic proinflammatory state. ${ }^{70}$ This chronic inflammation is believed to cause the coronary microvascular endothelial cells to produce reactive oxygen species. Reactive oxygen species limit the bioavailability of nitric oxide, which decreases PKG. ${ }^{10}$ Low PKG levels have been associated with increased cardiomyocyte tension and hypertrophy. ${ }^{70}$ This leads to concentric LV remodeling and increased collagen deposition, LVDD, and HFpEF. ${ }^{10}$ This new paradigm proposed possible therapeutic strategies that should aim at treating endothelial dysfunction by reducing inflammation, nitric oxide donors, phosphodiesterase- 5 inhibitors, and the antioxidative properties of statins. ${ }^{10}$

Although there is a paucity of current data for the management of HFpEF, there are several promising therapies under investigation. A meta-analysis of 11 studies and almost 18,000 patients with HFpEF showed a trend of reduction in mortality rates in statin users; additional RCTs are needed. ${ }^{62}$ The most promising drug therapy is the angiotensin-receptor neprilysin inhibitor LCZ696. PARAMOUNT was a phase-II, multicenter trial comparing the change in NT-proBNP in HFpEF patients treated with LCZ696 compared with treatment with valsartan and resulted in a further reduction of NT-proBNP in the LCZ696 group. ${ }^{71}$ This now is being tested in the phase III trial PARAGON-HF (Efficacy and Safety of LCZ696 compared to Valsartan on Morbidity and Mortality in HF Patients with Preserved Ejection Fraction study). ${ }^{70}$ Elevated heart rate was found to be an independent predictor of adverse clinical outcomes; each standard deviation (12.4 bpm) increase in heart rate was associated with a $13 \%$ increased risk of cardiovascular death or hospitalization for $\mathrm{HF}^{72}$ As such, rate control has been analyzed using treatments such as vagal and carotid artery stimulation in the IPreserve Trial (irbesartan patients with HF and preserved systolic function) ${ }^{70}$ Finally, promise has been shown using wireless pulmonary artery pressure monitoring to guide management and reduce decompensation in HFpEF patients. In the prospective, singleblinded, randomized CardioMEMS Heart Sensor Allows Monitoring of Pressure to Improve Outcomes in NYHA (CHAMPION) Class III HF Patients trial, 119 patients were assigned randomly to either standard treatment or treatment guided by the implanted pressure sensor. After an average of 18 months of follow-up, the hemodynamically guided treatment group was $50 \%$ less likely to be hospitalized for $\mathrm{HF}^{73}$ These studies may have implications for perioperative physicians who often are asked to guide perioperative loading conditions in patients with HFpEF.

\section{Conclusion}

Identifying perioperative patients with $\mathrm{HFpEF}$ can be challenging for anesthesiologists because the diagnosis can be discrete. Guidance of fluid administration and medication management in this HF population are difficult due to changes in loading conditions that occur during the perioperative period. Proper perioperative risk stratification, optimization of 
comorbidities, and the ability to diagnose and manage worsening perioperative LVDD are all important factors to consider. Although substantial outcome data exist on the direct correlation of HFpEF to poor outcomes, significant gaps exist on perioperative treatment and the role anesthesiologists can play to improve outcomes in HFpEF patients. Future studies on preoperative optimization, intraoperative monitoring and management, and treatment of acute decompensation are warranted.

\section{Acknowledgments}

Dr. Shillcutt has received funding from the National Institute of Aging (1R03 AG045103-01A1), and is owner of Brave Enough, LLC, an educational leadership company.

\section{References}

1. Reddy YNG, Borlaug BA. Heart failure with preserved ejection fraction. Curr Probl Cardiol. 2016; 41:145-88. [PubMed: 26952248]

2. Borlaug BA. The pathophysiology of heart failure with preserved ejection fraction. Nat Rev Cardiol. 2014; 11:507-15. [PubMed: 24958077]

3. Heerebeek LV, Paulus WJ. Understanding heart failure with preserved ejection fraction: Where are we today? Neth Heart J. 2016; 24:227-36. [PubMed: 26909795]

4. Borlaug BA, Paulus WJ. Heart failure with preserved ejection fraction: Pathophysiology, diagnosis, and treatment. Eur Heart J. 2011; 32:670-9. [PubMed: 21138935]

5. Paulus WJ, Tschope C, Sanderson JE, et al. How to diagnose diastolic heart failure: A consensus statement on the diagnosis of heart failure with normal left ventricular ejection fraction by the Heart Failure and Echocardiography Associations of the European Society of Cardiology. Eur Heart J. 2007; 28:5239-50.

6. Abbate A, Arena R, Abouzaki N, et al. Heart failure with preserved ejection fraction: Refocusing on diastole. Int J Cardiol. 2015; 179:430-40. [PubMed: 25465302]

7. Ferrari R, Bohm M, Cleland JGF, et al. Heart failure with preserved ejection fraction: Uncertainties and dilemmas. Eur J Heart Fail. 2015; 17:665-71. [PubMed: 26079097]

8. Brouwers FP, de Boer RA, van der Harst P, et al. Incidence and epidemiology of new onset heart failure with preserved vs. reduced ejection fraction in a community-based cohort: 11-year follow-up of PREVEND. Eur Heart J. 2013; 34:1424-31. [PubMed: 23470495]

9. Samson R, Jaiswal A, Ennezat PV, et al. Clinical phenotypes in heart failure with preserved ejection fraction. J Am Heart Assoc. 2016; 5:1-15.

10. Paulus WJ, Tschöpe C. A novel paradigm for heart failure with preserved ejection fraction: Comorbidities drive myocardial dysfunction and remodeling through coronary microvascular endothelial inflammation. J Am Coll Cardiol. 2013; 62:263-71. [PubMed: 23684677]

11. Franssen C, Chen S, Unger A, et al. Myocardial microvascular inflammatory endothelial activation in heart failure with preserved ejection fraction. JACC Heart Failure. 2016; 4:312-24. [PubMed: 26682792]

12. Gladden JD, Linke WA, Redfield MM. Heart failure with preserved ejection fraction. Pflugers Arch. 2014; 466:1037-53. [PubMed: 24663384]

13. Zile MR, Baicu CF, Ikonomidis JS, et al. Myocardial stiffness in patients with heart failure and a preserved ejection fraction: Contributions of collagen and titin. Circulation. 2015; 131:1247-59. [PubMed: 25637629]

14. Toledo C, Andrade D, Lucero C, et al. Cardiac diastolic and autonomic dysfunction are aggravated by central chemoreflex activation in heart failure with preserved ejection fraction rats. J Physiol. 2017; 595:2479-95. [PubMed: 28181258]

15. Redfield MM. Heart failure preserved ejection fraction. N Engl J Med. 2016; 375:1868-77. [PubMed: 27959663] 
16. Sastry P, Theologou T, Field M, et al. Predictive accuracy of EuroSCORE: Is end-diastolic dysfunction a missing variable? Eur J Cardiothorac Surg. 2010; 37:261-6. [PubMed: 19773181]

17. Matyal R, Hess P, Subramaniam B, et al. Perioperative diastolic dysfunction during vascular surgery and its association with postoperative outcome. J Vasc Surgery. 2009; 50:70-6.

18. Salem R, Denault A, Couture P, et al. Left ventricular end-diastolic pressure is a predictor of mortality in cardiac surgery independently of left ventricular ejection fraction. Br J Anaesth. 2006; 97:292-7. [PubMed: 16835254]

19. Morello L, Riesle E, Alburquerque J, et al. Risk scores do not predict high mortality after coronary artery bypass surgery in the presence of diastolic dysfunction. Ann Thorac Surg. 2008; 85:124755. [PubMed: 18355505]

20. Liu J, Tanaka N, Murata K, et al. Prognostic value of pseudonormal and restrictive filling patterns on left ventricular remodeling and cardiac events after coronary artery bypass grafting. Am J Cardiol. 2003; 91:550-4. [PubMed: 12615258]

21. Kaw R, Hernandez A, Pasupuleti V, et al. Effect of diastolic dysfunction on postoperative outcomes after cardiovascular surgery: A systematic review and meta-analysis. J Thorac Cardiovasc Surg. 2016; 152:1142-53. [PubMed: 27364601]

22. Fayad A, Ansari M, Yang H, et al. Perioperative diastolic dysfunction in patients undergoing noncardiac surgery is an independent risk factor for cardiovascular events: A systematic review and meta-analysis. Anesth. 2016; 125:72-91.

23. Afilalo J, Flynn A, Shimony A, et al. Incremental value of the preoperative echocardiogram to predict mortality and major morbidity in coronary artery bypass surgery. Circulation. 2013; 127:356-64. [PubMed: 23239840]

24. Bernard F, Denault A, Babin D, et al. Diastolic dysfunction is predictive of difficult weaning from cardiopulmonary bypass. Anesth Analg. 2001; 92:291-8. [PubMed: 11159219]

25. Groban L, Sanders D, Houle T, et al. Prognostic value of tissue Doppler-derived E/e' on early morbid events after cardiac surgery. Echocardiography. 2010; 27:131-8. [PubMed: 20380676]

26. Marui A, Nishiwaki N, Komiya T, et al. Comparison of 5-year outcomes after coronary artery bypass grafting in heart failure patients with versus without preserved left ventricular ejection fraction (from the CREDO-Kyoto CABG Registry Cohort-2). Am J Cardiol. 2015; 116:580-6. [PubMed: 26092271]

27. Dalén M, Lund LH, Ivert T, et al. Survival after coronary artery bypass grafting in patients with preoperative heart failure and preserved vs reduced ejection fraction. JAMA Cardiol. 2016; 1:530 8. [PubMed: 27434816]

28. Cabrera Schulmeyer M, Arriaza N. Good prognostic value of the intraoperative tissue Dopplerderived index E/e' after non-cardiac surgery. Min Anest. 2012; 78:1013-8.

29. Cho D, Park S, Kim M, et al. Presence of preoperative diastolic dysfunction predicts postoperative pulmonary edema and cardiovascular complications in patients undergoing noncardiac surgery. Echocardiography. 2014; 31:42-9. [PubMed: 23919453]

30. Denault A, Couture P, Buithieu J, et al. Left and right ventricular diastolic dysfunction as predictors of difficult separation from cardiopulmonary bypass. Can J Anesth. 2006; 53:1020-9. [PubMed: 16987858]

31. Fayad A, Yang H, Nathan H, et al. Acute diastolic dysfunction in thoracoabdominal aortic anuerysm surgery. Can J Anaesth. 2006; 52:168-73.

32. Oktay AA, Shah SJ. Diagnosis and management of heart failure with preserved ejection fraction: 10 key lessons. Curr Cardiol Rev. 2015; 11:42-52. [PubMed: 24251461]

33. Yancy CW, Jessup M, Bozkurt B, et al. 2013 ACCF/AHA guideline for the management of heart failure: A report of the American College of Cardiology Foundation/American Heart Association Task Force on Practice Guidelines. J Am Coll Cardiol. 2013; 62:147-239.

34. Ponikowski P, Voors AA, Anker SD, et al. 2016 ESC guidelines for the diagnosis and treatment of acute and chronic heart failure. Eur Heart J. 2016; 37:2129-200. [PubMed: 27206819]

35. Hernandez AF, Whellan DJ, Stroud S, et al. Outcomes in heart failure patients after major noncardiac surgery. J Am Coll Cardiol. 2004; 44:1446-53. [PubMed: 15464326]

36. Phillip B, Pastor D, Bellows W, et al. The prevalence of preoperative diastolic filling abnormalities in geriatric surgical patients. Anesth Analg. 2003:1214-21. 
37. Nagueh SF, Appleton CP, Gillebert TC, et al. Recommendations for the evaluation of left ventricular diastolic function by echocardiography. J Am Soc Echocardiogr. 2009; 22:107-33. [PubMed: 19187853]

38. Swaminathan M, Nicoara A, Phillips-Bute BG, et al. Utility of a simple algorithm to grade diastolic dysfunction and predict outcome after coronary artery bypass graft surgery. Ann Thorac Surg. 2011; 91:1844-50. [PubMed: 21492828]

39. Penicka M, Vanderheyden M, Bartunek J. Diagnosis of heart failure with preserved ejection fraction: Role of clinical Doppler echocardiography. Heart. 2013; 100:68-76. [PubMed: 23543282]

40. Adeniran I, MacIver DH, Hancox JC, et al. Abnormal calcium homeostasis in heart failure with preserved ejection fraction is related to both reduced contractile function and incomplete relaxation: An electromechanically detailed biophysical modeling study. Front Physiol. 2015; 6:78. [PubMed: 25852567]

41. Hettrick D, Pagel P, Warltier D. Desflurane, sevoflurane, and isoflurane impair canine left ventricular-arterial coupling and mechanical efficiency. Anesthesiology. 1996; 85:403-13. [PubMed: 8712457]

42. Hettrick D, Pagel P, Warltier D. Alterations in canine left ventricular-arterial coupling and mechanical efficiency produced by propofol. Anesthesiology. 1997; 86:1088-93. [PubMed: 9158358]

43. Pagel P, Kehl F, Gare M, et al. Mechanical function of the left atrium: New insights based on analysis of pressure-volume relations and Doppler echocardiography. Anesthesiology. 2003; 98:975-94. [PubMed: 12657862]

44. McIlroy DR, Lin E, Durkin C. Intraoperative transesophageal echocardiography: A critical appraisal of its current role in the assessment of diastolic dysfunction. J Cardiothorac Vasc Anesth. 2015; 29:1033-43. [PubMed: 25979529]

45. Matyal R, Skubas NJ, Shernan SK, et al. Perioperative assessment of diastolic dysfunction. Anesth Analg. 2011; 113:449-72. [PubMed: 21813627]

46. Mahmood F, Jainandunsing J, Matyal R. A practical approach to echocardiographic assessment of perioperative diastolic dysfunction. J Cardiothorac Vasc Anesth. 2012; 26:1115-23. [PubMed: 23122300]

47. Shillcutt SK, Montzingo CR, Agrawal A, et al. Echocardiography-based hemodynamic management of left ventricular diastolic dysfunction: A feasibility and safety study. Echocardiography. 2014; 31:1189-98. [PubMed: 24660834]

48. Meijers WC, van der Velde AR, de Boer RA. Biomarkers in heart failure with preserved ejection fraction. Neth Heart J. 2016; 24:252-8. [PubMed: 26942916]

49. Shimiaie J, Sherez J, Aviram G, et al. Determinants of effort intolerance in patients with heart failure: Combined echocardiography and cardiopulmonary stress protocol. JACC Heart Failure. 2015; 3:803-14. [PubMed: 26449998]

50. Nagueh S, Smiseth O, Appleton C, et al. Recommendations for the evaluation of left ventricular diastolic function by echocardiography: An update from the American Society of Echocardiography and the European Association of Cardiovascular Imaging. J Am Soc Echocardiogr. 2016; 29:277-314. [PubMed: 27037982]

51. Ha J-W, Oh JK, Pellikka PA, et al. Diastolic stress echocardiography: A novel noninvasive diagnostic test for diastolic dysfunction using supine bicycle exercise Doppler echocardiography. $\mathrm{J}$ Am Soc Echocardiogr. 2005; 18:63-8. [PubMed: 15637491]

52. Obokata M, Kane G, Reddy Y, et al. Role of diastolic stress testing in the evaluation for heart failure with preserved ejection fraction: A simultaneous invasive-echocardiographic study. Circulation. 2017; 135:825-38. [PubMed: 28039229]

53. Gandhi PU, Gaggin HK, Redfield MM, et al. Insulin-like growth factor-binding protein-7 as a biomarker of diastolic dysfunction and functional capacity in heart failure with preserved ejection fraction: Results from the RELAX Trial. JACC Heart Failure. 2016; 4:860-9. [PubMed: 27744089]

54. Kotecha D, Lam CSP, van Veldhuisen DJ, et al. Heart failure with preserved ejection fraction and atrial fibrillation: Vicious twins. J Am Coll Cardiol. 2016; 68:2217-28. [PubMed: 27855811] 
55. Lam CSP, Rienstra M, Tay WT, et al. Atrial fibrillation in heart failure with preserved ejection fraction: Association with exercise capacity, left ventricular filling pressures, natriuretic peptides, and left atrial volume. JACC Heart Failure. 2016; 5:92-8. [PubMed: 28017355]

56. Carbone S, Canada JM, Buckley LF, et al. Obesity contributes to exercise intolerance in heart failure with preserved ejection fraction. J Am Coll Cardiol. 2016; 68:2487-8. [PubMed: 27908355]

57. Anjan VY, Loftus TM, Burke MA, et al. Prevalence, clinical phenotype, and outcomes associated with normal B-type natriuretic peptide levels in heart failure with preserved ejection fraction. Am J Cardiol. 2012; 110:870-6. [PubMed: 22681864]

58. Bishu K, Deswal A, Chen HH, et al. Biomarkers in acutely decompensated heart failure with preserved or reduced ejection fraction. Am Heart J. 2012; 164:763-70. [PubMed: 23137508]

59. Fleisher LA, Fleischmann KE, Auerbach AD, et al. 2014 ACC/AHA guideline on perioperative cardiovascular evaluation and management of patients undergoing noncardiac surgery: A report of the American College of Cardiology/American Heart Association Task Force on practice guidelines. J Am Coll Cardiol. 2014; 64:e77-178. [PubMed: 25091544]

60. Alsaddique A, Royse A, Royse C, et al. Management of diastolic heart failure following cardiac surgery. Eur J Cardiothorac Surg. 2009; 35:241-9. [PubMed: 19084422]

61. Butler J, Fonarow G, Zile M, et al. Developing therapies for heart failure with preserved ejection fraction: Current state and future directions. JACC Heart Fail. 2014; 2:97-112. [PubMed: 24720916]

62. Liu G, Zheng X, Xu Y, et al. Meta-analysis of the effect of statins on mortality in patients with preserved ejection fraction. Am J Cardiol. 2014; 113:1198-204. [PubMed: 24513478]

63. Shah R, Desai A, Givertz M. The effect of renin-angiotensin system inhibitors on mortality and heart failure hospitalization in patients with heart failure and preserved ejection fraction: A systematic review and meta-analysis. J Cardiac Fail. 2010; 16:260-7.

64. Pitt B, Pfeffer M, Assmann S, et al. Spironolactone for heart failure with preserved ejection fraction. N Engl J Med. 2014; 370:1383-92. [PubMed: 24716680]

65. Bavishi C, Chatterjee S, Anther S, et al. Beta-blockers in heart failure with preserved ejection fraction: A meta-analysis. Heart Fail Rev. 2015; 20:193-201. [PubMed: 25034701]

66. Redfield M, Chen H, Borlaug B, et al. Effect of phosphodiesterase-5 inhibition on exercise capacity and clinical status in heart failure with preserved ejection fraction. J Am Med Assoc. 2013; 309:1268-77.

67. McMurray J, Adamopoulos S, Anker S, et al. ESC guidelines for the diagnosis and treatment of acute and chronic heart failure 2012. Eur Heart J. 2012; 33:1787-847. [PubMed: 22611136]

68. Münzel T, Gori T, Keaney J, et al. Pathophysiological role of oxidative stress in systolic and diastolic heart failure and its therapeutic implications. Eur Heart J. 2015; 36:2555-64. [PubMed: 26142467]

69. Dieberg G, Ismail H, Giallauria F, et al. Clinical outcomes and cardiovascular responses to exercise training in heart failure patients with preserved ejection fraction: A systematic review and metaanalysis. J Appl Physiol. 2015; 119:726-33. [PubMed: 25749444]

70. Heerebeek L, Hamdani N, Falcáo-Pires, et al. Low myocardial protein kinase G activity in heart failure with preserved ejection fraction. Circulation. 2012; 126:830-9. [PubMed: 22806632]

71. Solomon S, Zile M, Pieske B, et al. The angiotensin receptor neprilysin inhibitor LCZ696 in heart failure with preserved ejection fraction: A phase 2 double-blind randomized controlled trial. Lancet. 2012; 380:1387-95. [PubMed: 22932717]

72. Böhm M, Perez A, Jhund P, et al. Relationship between heart rate and mortality and morbidity in the irbesartan with heart failure and preserved systolic function trial (I-Preserve). Eur J Heart Fail. 2014; 16:778-87. [PubMed: 24864045]

73. Adamson P, Abraham W, Bourge R, et al. Wireless pulmonary artery pressure monitoring guides management to reduce decompensation in heart failure with preserved ejection fraction. Circ Heart Fail. 2014; 7:935-44. [PubMed: 25286913]

74. Flu W, van Kujik J, Hoeks S, et al. Prognostic Implications of Asymptomatic Left Ventricular Dysfunction in Patients Undergoing Vascular Surgery. Anesthesiology. 2010; 112:1316-24. [PubMed: 20502115] 


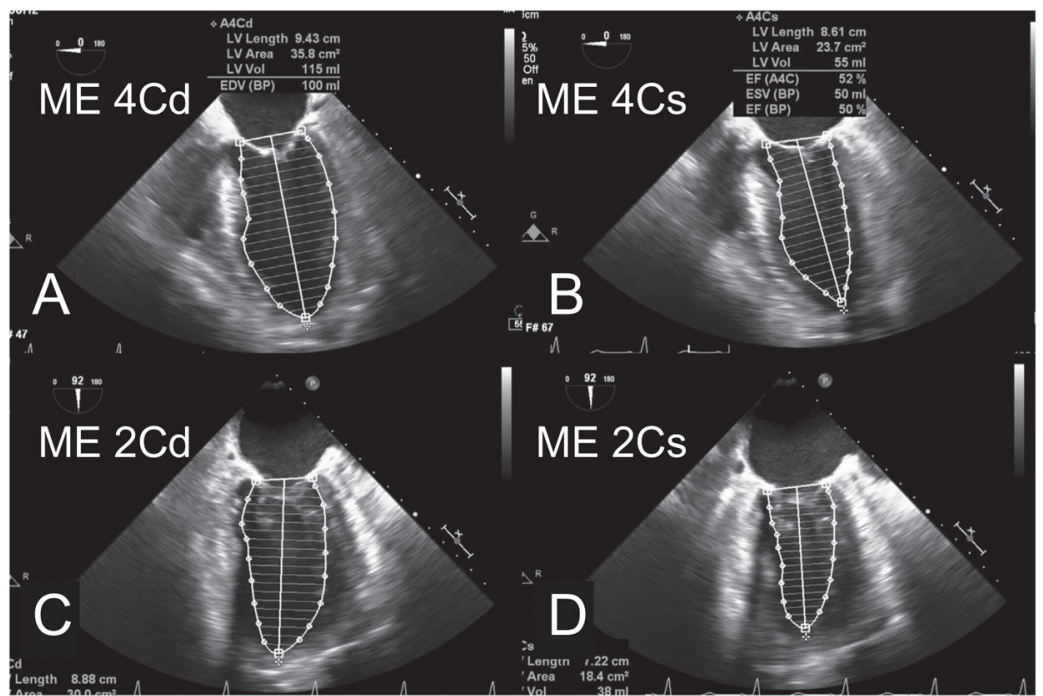

Fig 1.

The biplane method of measuring LVEF. (A) The midesophageal 4-chamber (ME 4C) view at 0 degrees during diastole $(M E 4 C d)$ in which the endocardial boarder is traced. (B) The border is traced with the $\mathrm{ME} 4 \mathrm{C}$ view during systole $(M E 4 C S)$. (C) The second view for the biplane method is the midesophageal 2-chamber (ME 2C) view at 90 degrees. The $\mathrm{ME} 2 \mathrm{C}$ view during diastole $(M E 2 C d$ ) in which the endocardium is traced. (D) The ME $2 \mathrm{C}$ view during systole (ME 2CS) with the endocardium traced. The end diastolic volume of the left ventricle can be seen (A) with a volume of $100 \mathrm{~mL}$, which with a body surface area of 1.6 is $63 \mathrm{~mL} / \mathrm{m}^{2}$. The biplane EF (B) measured 50\%. 

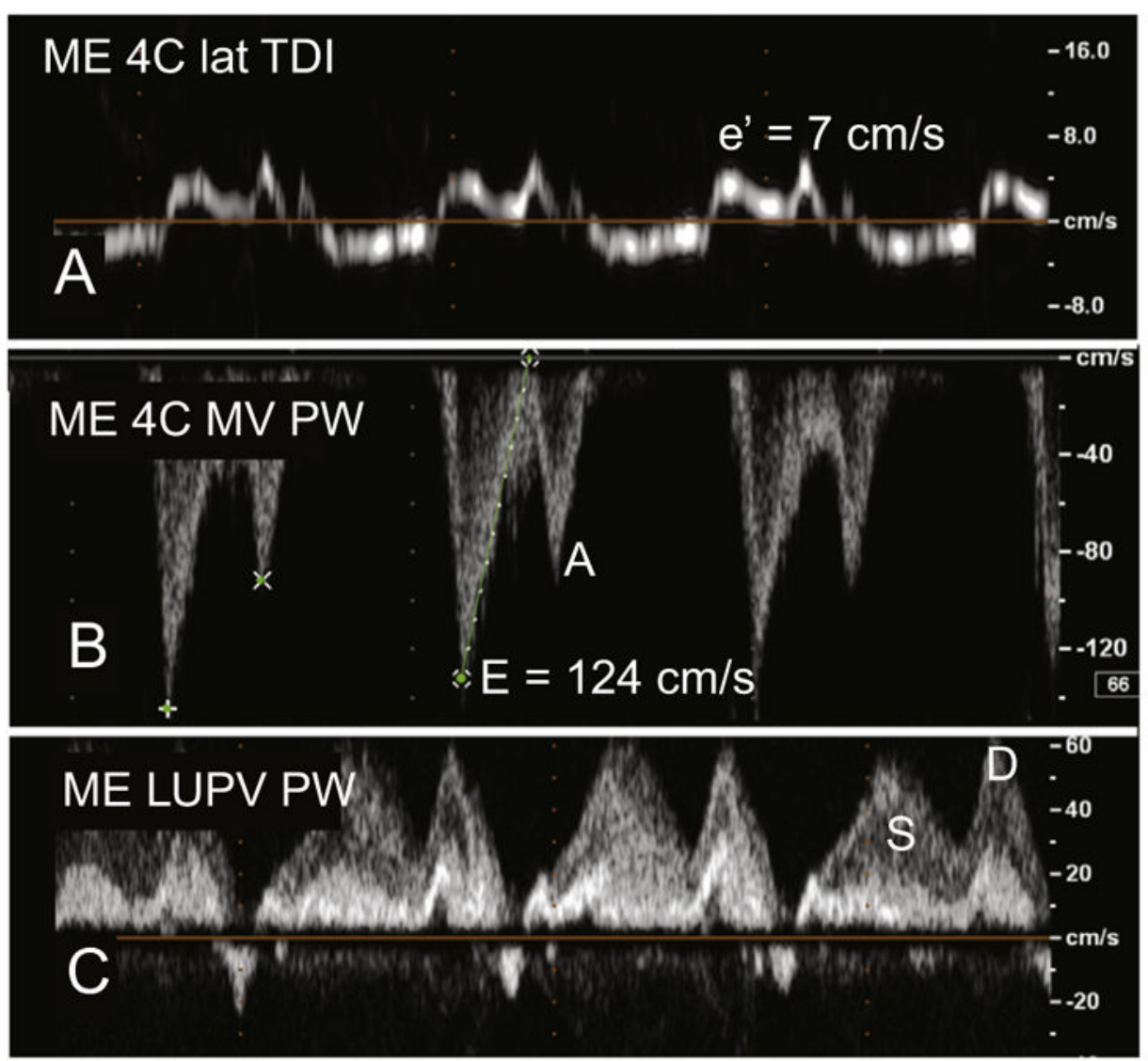

Fig 2.

The Doppler evaluation of diastolic filling. (A) The tissue Doppler velocities of the lateral mitral annulus are seen measuring $7 \mathrm{~cm} / \mathrm{sec}$, which is below the threshold of normal $(\geq 10$ $\mathrm{cm} / \mathrm{sec}$ ), indicating diastolic dysfunction. (B) To further verify the presence of diastolic dysfunction and the presence of elevated diastolic filling pressures, pulse-wave Doppler measurements of mitral inflow are demonstrated. Here the E-wave velocity is seen to be 125 $\mathrm{cm} / \mathrm{sec}$ and larger than the $\mathrm{A}$ wave. This gives an $\mathrm{E} / \mathrm{e}^{\prime}$ ratio of 18 (>13), and the E:A ratio is $>1$. In addition, the E-wave velocity is $>100 \mathrm{~cm} / \mathrm{sec}$. Even though not sensitive for increased filling pressures, it is specific. (C) The pulmonary venous flow measured using pulse-wave Doppler is seen. In this example, despite evidence of significantly elevated diastolic filling pressures, the pulmonary venous flow appears codominant, demonstrating how pulmonary venous flow can be nonspecific in the setting of diastolic dysfunction. 


\section{Table 1}

Characteristics of $\mathrm{HFpEF}^{1,3,4,5,7,15}$

\begin{tabular}{|c|c|c|}
\hline HFpEF & & HFrEF \\
\hline \multicolumn{3}{|l|}{ Echocardiographic findings } \\
\hline Diastolic dysfunction & Very prevalent finding-impaired left heart filling & Common but not diagnostic \\
\hline Tissue Doppler & Reduced myocardial tissue Doppler velocities & Reduced tissue Doppler velocities \\
\hline Left ventricle dimensions & Normal LV chamber size & Dilated LV chamber size \\
\hline Left ventricle wall thickness ${ }^{5}$ & Increased wall thickness (concentric remodeling) ${ }^{5}$ & Lower LV mass:volume ratio ${ }^{5}$ \\
\hline $\mathrm{EF}$ & Normal (>50\%) & Reduced $(<30 \%)$ \\
\hline \multicolumn{3}{|l|}{ Physiologic findings } \\
\hline Pressure-volume loops ${ }^{5}$ & $\begin{array}{l}\text { Pressure-volume loops show an upward or leftward } \\
\text { shift of the LV end-systolic pressure volume } \\
\text { relationship, reflecting increased myocardial stiffness }\end{array}$ & $\begin{array}{l}\text { Decreased myocardial contractility } \\
\text { (rightward and downward) })^{5}\end{array}$ \\
\hline Vascular changes & Ventriculoarterial stiffening & Less ventriculoarterial stiffening \\
\hline Loading conditions ${ }^{15}$ & $\begin{array}{l}\text { Greater blood pressure variability from changes in } \\
\text { loading conditions_-both preload and afterload }{ }^{15}\end{array}$ & $\begin{array}{l}\text { Afterload reduction actually increases stroke } \\
\text { volume }\end{array}$ \\
\hline Wall tension and BNP release $\mathrm{e}^{4}$ & Increased wall thickness and lower and $\mathrm{BNP}^{4}$ & High BNP levels \\
\hline Structural alterations ${ }^{3}$ & $\begin{array}{l}\text { Cardiomyocyte hypertrophy and myocardial interstitial } \\
\text { fibrosis }^{3}\end{array}$ & Eccentric remodeling \\
\hline \multicolumn{3}{|l|}{$\begin{array}{l}\text { Clinical differences and } \\
\text { comorbidities }\end{array}$} \\
\hline Age & Older median age & Younger median age \\
\hline Sex & More likely to be female & No sex predilection \\
\hline Hypertension & Very high prevalence of systolic hypertension & Not associated with hypertension \\
\hline Medical comorbidities $^{1}$ & $\begin{array}{l}\text { Higher frequency of obesity, diabetes mellitus, } \\
\text { metabolic syndrome, renal dysfunction, anemia, and } \\
\text { atrial fibrillation }^{1}\end{array}$ & Less prevalent ${ }^{1}$ \\
\hline 5 -year mortality ${ }^{7}$ & $20 \%-40 \% 7$ & $30 \%-50 \% 7$ \\
\hline
\end{tabular}

Abbreviations: BNP, brain natriuretic peptide; HFpEF, heart failure with preserved ejection fraction; HFrEF, heart failure with reduced ejection fraction; LV, left ventricular. 


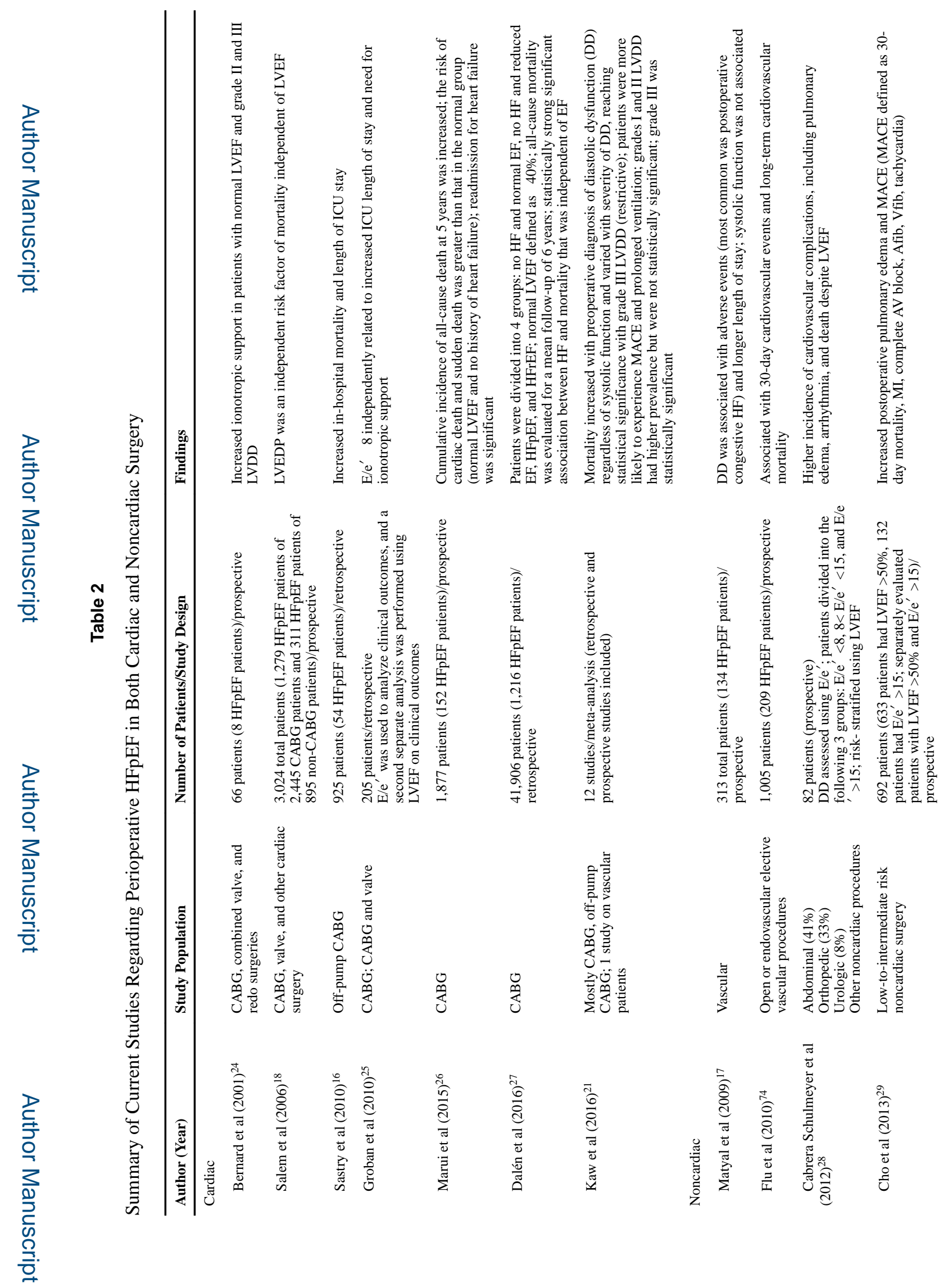

J Cardiothorac Vasc Anesth. Author manuscript; available in PMC 2018 October 01. 


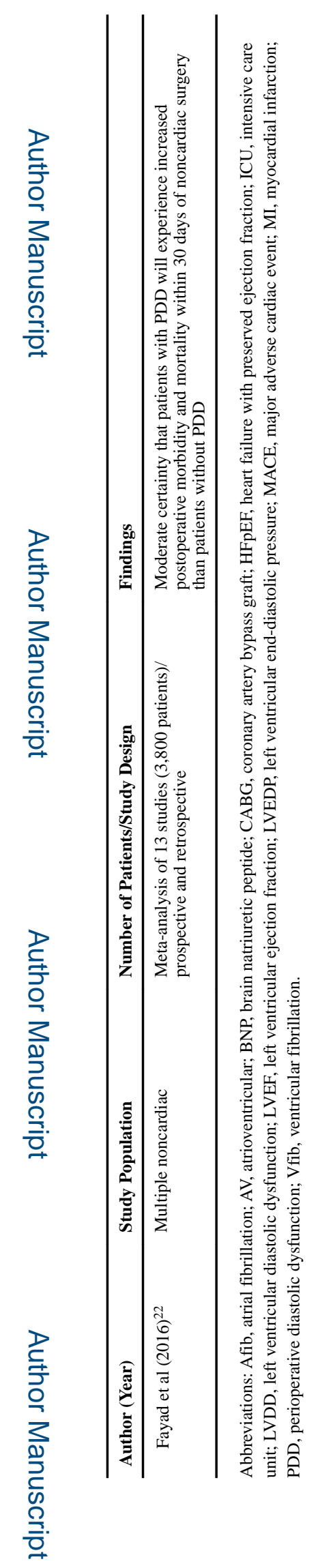


Table 3

Common Clinical Symptoms Reported by Patients With HF and Standard Descriptive Nomenclature for HF Severity

\begin{tabular}{|c|c|}
\hline \multicolumn{2}{|c|}{ Symptoms of HF } \\
\hline \multicolumn{2}{|l|}{ Fatigue } \\
\hline \multicolumn{2}{|c|}{ Shortness of breath } \\
\hline \multicolumn{2}{|l|}{ Dyspnea } \\
\hline \multicolumn{2}{|c|}{ Dyspnea on exertion } \\
\hline \multicolumn{2}{|c|}{ Ankle swelling } \\
\hline \multicolumn{2}{|c|}{ Breathlessness } \\
\hline \multicolumn{2}{|c|}{ Nocturnal dyspnea } \\
\hline \multicolumn{2}{|c|}{ Orthopnea } \\
\hline \multicolumn{2}{|c|}{ Dizziness } \\
\hline \multicolumn{2}{|c|}{ ACC/AHA stages of HF } \\
\hline Stage A & At high risk for HF but without structural heart disease or symptoms of HF \\
\hline Stage B & Structural heart disease but without signs or symptoms of HF \\
\hline Stage C & Structural heart disease with prior or current symptoms of HF \\
\hline Stage D & Refractory HF requiring specialized interventions \\
\hline \multicolumn{2}{|c|}{ NYHA functional classifications } \\
\hline Class 1 & No limitations of physical activity; ordinary physical activity does not cause symptoms of HF \\
\hline Class 2 & Slight limitation of physical activity; comfortable at rest but ordinary physical activity results in symptoms of HF \\
\hline Class 3 & Marked limitations in physical activity; comfortable at rest but less-than-ordinary physical activity causes symptoms of HF \\
\hline Class 4 & Unable to carry on any physical activity without symptoms of $\mathrm{HF}$ or symptoms of $\mathrm{HF}$ at rest \\
\hline
\end{tabular}

NOTE: Adapted from Oktay and Shah. ${ }^{32}$

Abbreviations: ACC/AHA, American College of Cardiologists and American Heart Association; HF, heart failture; NYHA, New York Heart Association. 


\section{Table 4}

Definitions and Criteria for the Diagnosis of HF With Preserved EF by the American College of Cardiologists and American Heart Association and the $\mathrm{ESC}^{34}$

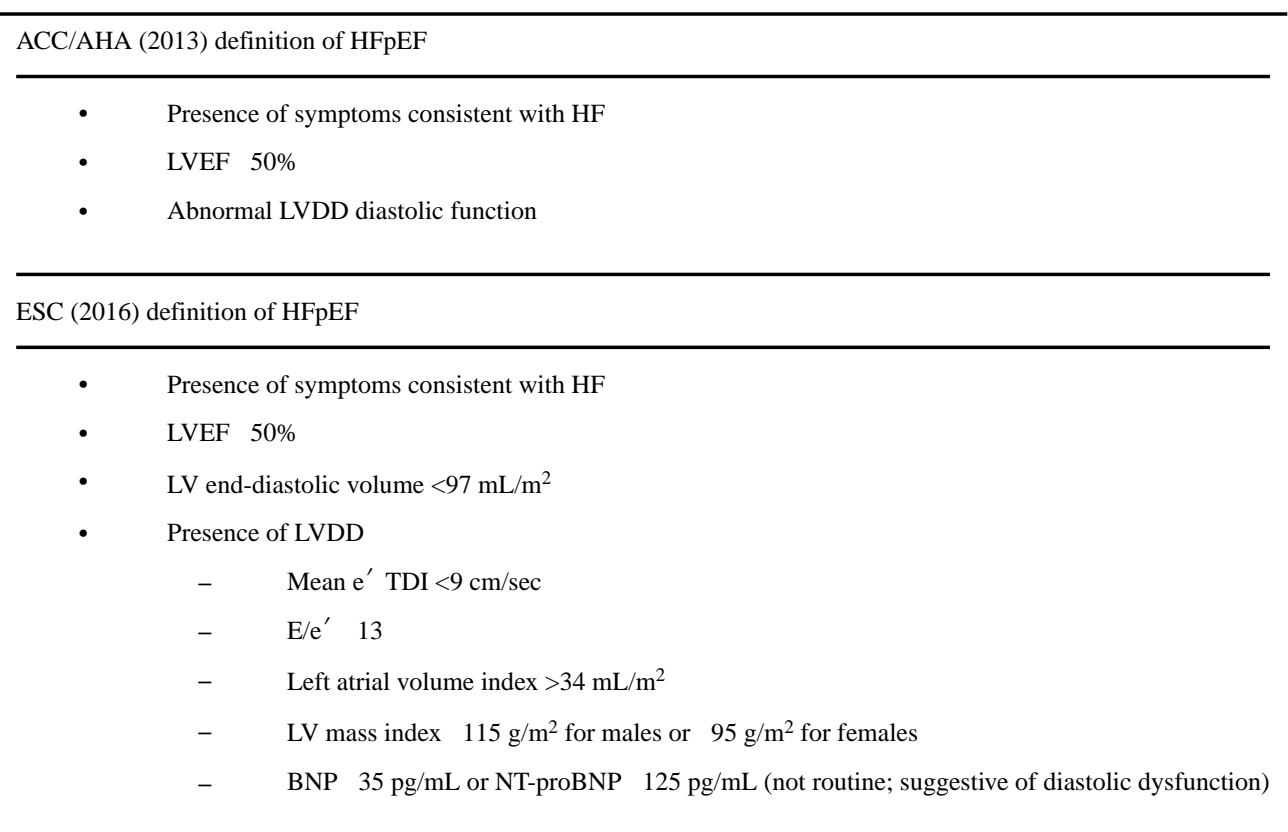

Abbreviation: ACC/AHA, American College of Cardiologists and American Heart Association; ESC, European Society of Cardiology; HFpEF, heart failure with preserved ejection fraction; LVDD, left ventricular diastolic dysfunction; LVEF, left ventricular ejection fraction; TDI, tissue Doppler imaging. 
Table 5

Criteria Used for Intraoperative Classification of Diastolic Function

\begin{tabular}{lcccc}
\hline & Lat TDI e $^{\prime}$ & E/A & E/e $^{\prime}$ & Pulmonary Veins \\
\hline Normal function & $\geq 10 \mathrm{~cm} / \mathrm{sec}$ & $>1$ & $\$ 8$ & $\mathrm{~S}>\mathrm{D}$ \\
Impaired relaxation & $<10 \mathrm{~cm} / \mathrm{sec}$ & $<1$ & $9-12$ & $\mathrm{~S}>\mathrm{D}$ \\
Elevated filling pressures (pseudonormal) (Restrictive filling) & $<10 \mathrm{~cm} / \mathrm{sec}$ & $>1$ & $\geq 13 \mathrm{~S}$ & $<$ D systolic blunting \\
\hline
\end{tabular}

Abbreviations: D, diastolic; S, systolic; TDI, tissue Doppler imaging. 


\section{Table 6}

Perioperative Management Goals for HFpEF patients ${ }^{47,60,61,63,64,67,70-72}$

\begin{tabular}{|c|c|c|}
\hline \multicolumn{3}{|c|}{ Clinical Goals for the Perioperative Management of HFPEF } \\
\hline \multicolumn{3}{|l|}{ What is known ${ }^{47,61}$} \\
\hline \multirow[t]{4}{*}{ Management strategies } & • & LVDD is an independent risk factor for perioperative cardiovascular events \\
\hline & • & Intraoperative echocardiography is the best tool to assess diastolic dysfunction \\
\hline & • & $\begin{array}{l}\text { Echocardiographically-guided hemodynamic management may be a safe alternative } \\
\text { standard management }\end{array}$ \\
\hline & • & $\begin{array}{l}\text { Elevated heart rate in HFpEF patients is associated with increased risk of } \\
\text { cardiovascular death and HF hospitalization }\end{array}$ \\
\hline \multirow[t]{6}{*}{ Comorbid conditions Optimization ${ }^{60,61}$} & $\bullet$ & Aggressive control of chronically uncontrolled hypertension \\
\hline & • & Tight glycemic control for diabetes mellitus \\
\hline & • & Maintain normal sinus rhythm \\
\hline & • & $\begin{array}{l}\text { Rate control patients with chronic atrial fibrillation (verapamil, diltiazem, or beta- } \\
\text { blocker) }\end{array}$ \\
\hline & • & CPAP devices for patients with OSA \\
\hline & • & Avoid fluid retention \\
\hline \multirow[t]{3}{*}{ Treatments to avoid ${ }^{63,67}$} & - & Thiazolidinediones (glitazones) \\
\hline & • & NSAIDs and COX-2 inhibitors (may cause sodium and water retention) \\
\hline & • & $\mathrm{ARB}$ in combination with $\mathrm{ACE}$ inhibitor and mineralocorticoid antagonist \\
\hline \multicolumn{3}{|l|}{ What is not known $62-64,67,70-72$} \\
\hline \multirow[t]{2}{*}{ Treatments } & - & $\begin{array}{l}\text { The benefit of multiple traditional therapies of HF treatment: ACE-I/ARBs, statins, } \\
\text { beta-blocker }\end{array}$ \\
\hline & • & The role of angiotensin-receptor neprilysin inhibitors \\
\hline
\end{tabular}

Abbreviations: ACE, angiotensin-converting enzyme; ARB, angiotensin-receptor blocker; COX-2, cyclooxygenase 2; CPAP, continuous positive airway pressure; HFpEF, heart failure with preserved ejection fraction; LVDD, left ventricular diastolic dysfunction; NSAIDs, nonsteroidal antiinflammatory drugs; OSA, obstructive sleep apnea. 